\title{
Les bambous gravés kanak et Marguerite Dellenbach
}

\section{Christian Coiffier}

\section{(2) OpenEdition}

1 Journals

\section{Édition électronique}

URL : http://journals.openedition.org/jso/1912

DOI : $10.4000 /$ jso. 1912

ISSN : 1760-7256

\section{Éditeur}

Société des océanistes

\section{Édition imprimée}

Date de publication : 15 décembre 2008

Pagination : 320-325

ISBN : 978-2-85430-012-3

ISSN : 0300-953x

\section{Référence électronique}

Christian Coiffier, «Les bambous gravés kanak et Marguerite Dellenbach », Journal de la Société des Océanistes [En ligne], 126-127 | Année 2008, mis en ligne le 01 décembre 2008, consulté le 17 juin 2020. URL : http://journals.openedition.org/jso/1912 ; DOI : https://doi.org/10.4000/jso.1912 


\title{
Les bambous gravés kanak et Marguerite Dellenbach
}

par

\author{
Christian COIFFIER*
}

\section{Découverte d'un nouveau bambou gravé kanak}

L'ensemble des bambous gravés kanak connus dans le monde jusqu'à présent est de l'ordre de plus de 230 spécimens et aucun d'entre eux n'est similaire. Ce bambou, donné à l'association des Amis du musée de l'Homme, mesure $112 \mathrm{~cm}$ de longueur pour un diamètre de $5 \mathrm{~cm}$. Il présente trois nœuds et deux entre-nœuds. L'un de ceux-ci, en haut, est ouvert et l'autre, en bas, fermé avec cependant un petit trou en son centre. La position verticale correspond au sens de lecture de la majorité des dessins gravés sur ce bambou. L'état de conservation de ce dernier n'est pas excellent, dans la mesure ou l'entrenœud haut présente six fentes et celui du bas, quatre fentes. Nous lirons les différents registres de haut en bas.

Sur l'entre-nœud du haut, on distingue quatre hommes et deux femmes. Certains fument et portent des armes contondantes. En dessous sont visibles trois couples associés à six lézards dont trois traités de même façon, des dessins de chevrons sur le dos. Deux autres présentent des hachures sur le dos et le corps du dernier est juste recouvert de points. Un anneau avec des chevrons vient séparer le registre suivant. Une frise de quatre femmes européennes : deux de petite stature, vraisemblablement des jeunes filles, et deux de grande stature, leurs mères portent des robes à «faux-cul » ainsi qu'un chapeau avec un nœud de rubans pour l'une d'entre elles. Le sol est représenté par une simple ligne. Au-dessous, une frise de cinq hommes européens portant moustache, habillés d'un pantalon serré et d'une redingote. Chacun d'entre eux porte un chapeau. Un seul n'a pas de canne à la main. Le traitement de ces personnages européens est particulièrement soigné et réaliste.

Au-dessus du nœud situé au milieu se trouve un anneau avec des motifs en forme de rails de chemin de fer et, dessous, un autre anneau constitué de zigzags. L'entre-nœud bas est divisé également en deux par un anneau en forme de rails de chemin de fer. On aperçoit, en partie haute, une frise avec une série d'arbres divers dont le graphisme des feuillages est très esthétique. Au milieu d'entre eux, un homme et un oiseau sur le sol. Au-dessous, une mare avec des nénuphars, six canards et deux grenouilles. Au dos, se trouve représenté un enclos au sein duquel se trouvent vingt-deux hommes en armes. L'un d'entre eux fume ; un autre boit le contenu d'une gourde. Sur le registre bas, deux cases de chaque côté d'une place cérémonielle bordée de cocotiers et de pins colonnaires. Les édifices et les végétaux sont représentés rabattus suivant les canons du dessin kanak (Lobsiger-Dellenbach, 1965 : 271-287). Sur l'autre face se trouve une case avec, à sa base, deux arbres aux rameaux volubiles et aux feuilles trilobées. Des hommes porteurs de casse-tête, de branches d'arbre et de lancettes accompagnent cette scène champêtre. Certains hommes fument la pipe et une femme laisse apparaître ses parties génitales. Nous laissons aux spécialistes l'inter-

* Maître de conférences au MNMN, coiffier@mrhr.fr 


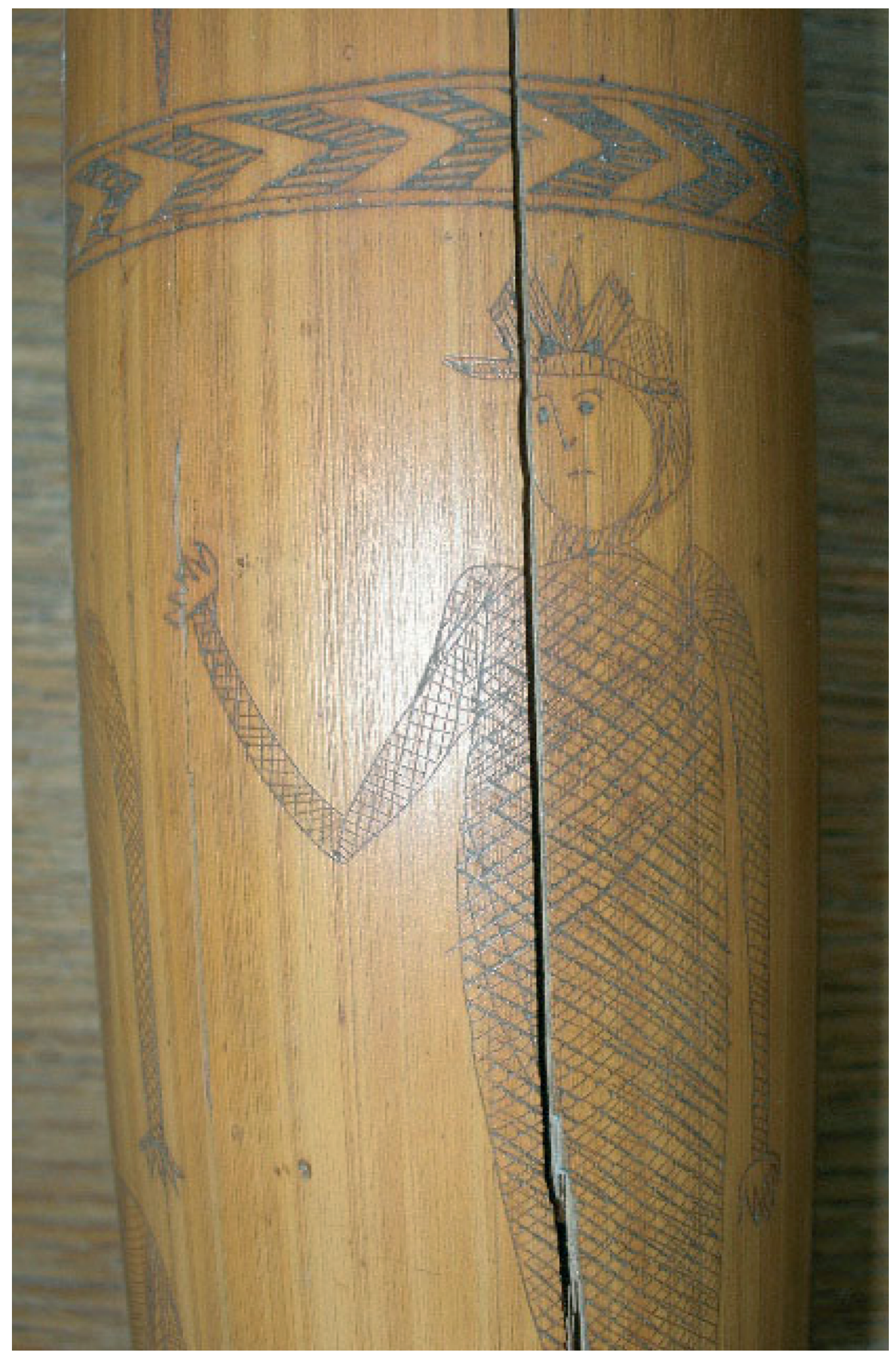

Рното 1. - Gros plan du buste d'une des femmes européennes gravée sur le bambou offert à la Société des amis du musée de l'Homme en 2008 
prétation de ces diverses scènes représentées sur ce bambou jusqu'alors inconnu.

\section{L'exposition Bambous kanak. Une passion de Marguerite Lobsiger-Dellenbach du musée d'Eth- nographie de Genève (MEG) ${ }^{1}$}

L'exposition a été préparée et présentée par ses commissaires, Roberta Colombo-Dughoud et Lorin Wüscher. Elle s'articule en trois salles contiguës du musée. La première évoque le travail du couple Marguerite et Georges LobsigerDellenbach. Sur deux écrans, des films présentant leur travail de recherche sur les bambous passent en boucle. Une table lumineuse permet de mettre en situation et d'expliquer leurs méthodes de travail pour réaliser les relevés des dessins sur les bambous. Les murs, et même une partie du plafond, sont recouverts de copies et d'originaux choisis parmi les centaines de relevés réalisés en plus de trente ans. La seconde salle expose la collection de bambous gravés kanak du musée de Genève dans une ambiance très sombre où chacune des pièces est éclairée par un spot. Les dessins d'un des bambous placé dans une vitrine sont particulièrement bien expliqués sur un cartel placé à bonne hauteur pour être lu sans difficulté. Les autres pièces sont exposées verticalement et parallèlement fixées sur des filins d'acier, ce qui en rend la lecture parfois un peu difficile. Chacune de ces pièces est commentée sur des cartels lumineux placés aux murs. La pénombre ne permet pas d'apprécier à leur juste valeur la grande qualité esthétique de certains dessins des bambous présentés. La troisième salle, beaucoup plus éclairée, présente les bambous récemment acquis par le musée auprès de l'artiste kanak Micheline Néporon. Les motifs dessinés évoquent les problèmes de la vie quotidienne actuelle en Nouvelle-Calédonie comme les dangers de l'alcoolisme, ce qui permet de comparer ces œuvres à celles du passé et d'y voir une forme de continuité.

C'est une très intéressante exposition qu'il faut visiter absolument, aussi bien pour les simples esthètes que pour ceux qui s'intéressent à l'art océanien. Un catalogue avec des articles de divers spécialistes et avec une excellente iconographie, complète cette exposition et permet d'en savoir plus sur la carrière et les méthodes de recherche du couple Lobsiger-Dellenbach, mais surtout d'apprécier un art très original et peu connu du grand public ( $c f$. présentation commentée du catalogue faite par Isabelle Leblic ci-dessus).

\section{Correspondance de Marguerite Dellenbach avec le musée de l'Homme au sujet des bambous gravés kanak}

L'inauguration de l'exposition Bambous kanak. Une passion de Marguerite LobsigerDellenbach qui se tient à Genève du 29 février 2008 au 4 janvier 2009 au MEG (musée d'Ethnographie de Genève) nous permet d'honorer à juste titre l'originale et passionnante recherche sur les bambous gravés kanak de l'ancienne directrice de ce musée, Marguerite LobsigerDellenbach. C'est l'occasion pour la Société des Océanistes, outre la présentation de l'exposition et de son catalogue, d'évoquer la correspondance que celle-ci a entretenue avec ses collègues du musée de l'Homme pour poursuivre ses travaux et pour pouvoir les publier. Le hasard a voulu que, dans les jours qui précédèrent notre visite à Genève, nous soyons contacté par Madame et Monsieur Bastard qui souhaitait faire don d'objets kanak anciens à la Société des Amis du musée de l'Homme. Parmi ces objets se trouvait un très intéressant bambou gravé que nous présenterons sommairement avec ces notes.

Les archives des courriers quotidiens de l'ancien département d'Océanie du musée de l'Homme recèlent une correspondance très intéressante qui permet de suivre l'évolution du travail de recherche de Marguerite Dellenbach sur les bambous gravés kanak de NouvelleCalédonie et sur sa carrière. Mariée en 1936 avec Georges Lobsiger (Colombo Dougoud, 2008 : 24), elle continuera cependant jusqu'en 1939 à être appelée mademoiselle Dellenbach et à signer ses courriers avec son nom de jeune-fille. La signature manuscrite avec les deux noms, Lobsiger-Dellenbach n'apparaît que sur une lettre de 1943 et c'est avec son pseudonyme Dell, donné par ses collègues du musée, qu'elle conclue la lettre de 1967. La signature, Marguerite $\mathrm{LD}$, utilisée dans sa dernière lettre de 1970 est la marque de sa relation amicale avec la conservatrice de l'époque, Françoise Girard.

Au début de l'année 1939, Marguerite Dellenbach, assistante du professeur Eugène Pittard, directeur du musée d'Ethnographie de Genève, écrit à Charles van den Broek d'Obrenan (lettre du 20 mars 1939) qui est chargé du département Océanie du musée de l'Homme depuis son retour du Pacifique en 1936 après vingt-six mois de circumnavigation autour du monde, avec ses quatre coéquipiers, sur son yacht La Korrigane (Van den Broek, 1939). Ce dernier a d'ailleurs rapporté deux bambous gravés de son séjour en 
Nouvelle-Calédonie (Coiffier, 2001: 100, 103). Elle le remercie de son amabilité lors de sa dernière visite à Paris pour lui avoir montré les collections rapportées de son voyage, mais elle lui exprime ses regrets de ne pas avoir eu le temps de voir les bambous néo-calédoniens se trouvant dans les vitrines et les réserves. Elle lui signale qu'elle vient de terminer l'étude des bambous gravés conservés à Bâle et à Berne et que ceux du musée Pigorini de Rome sont en cours d'étude. De plus, elle lui dit avoir publié, l'année passée, dans le Bulletin de la Société d'ethnographie de Paris son étude de six bambous du musée de $\mathrm{La}$ Rochelle (Dellenbach, 1938: 35-49). À cette époque, Marguerite Dellenbach est en voie de terminer sa thèse intitulée Bambous gravés de la Nouvelle Calédonie, leur interprétation ethnographique qu'elle soutiendra en 1940 à la faculté des Sciences économiques et sociales de l'université de Genève (Colombo Dougoud, 2008 : 18). Ne pouvant se rendre à Paris, elle lui propose de procéder, comme avec les autres musées cités précédemment, c'est à dire, de lui envoyer les bambous à Genève. Avec l'aide de son époux, elle réalisera très rapidement le relevé des dessins gravés sur les bambous avant de les retourner à leurs propriétaires. Les études détaillées se feront ensuite à partir de ces dessins. Selon son opinion, les études déjà réalisées sur certains bambous du musée de l'Homme par G. H. Luquet (1926) l'ont été d'un point de vue uniquement esthétique. Elle ne souhaite pas reprendre l'étude accomplie par M. Grünewald (1936) sur les trois bambous $\left(\mathrm{n}^{\text {os }} 35.103 .33,35.103 .32,35.103 .34\right)$ et déjà publié dans L'Anthropologie. Elle propose qu'on lui envoit les bambous par séries de deux ou trois à la fois. Charles van den Broek lui répond pour la remercier des tirés à part qu'elle lui a envoyés et pour la complimenter pour son travail qu'il trouve fort intéressant de même que son interprétation des différentes phases de la culture des ignames (lettre du 25 mars 1939). Dans une seconde lettre adressée trois jours après (lettre du 28 mars 1939), il lui fait part de l'autorisation du docteur Paul Rivet, directeur du musée de l'Homme, d'envoyer les bambous au musée de Genève à la seule condition que cette institution prenne en charge tous les frais de transport et d'assurances. Il propose qu'une première série de bambous se trouvant dans les réserves lui soit envoyée dès que ces conditions seront acceptées. Lorsque ces derniers auront été étudiés et renvoyés à Paris, alors une autre série se trouvant dans les vitrines pourra lui être expédiée. Il transmet également le désir de Paul Rivet d'obtenir pour la bibliothèque du musée de l'Homme l'ensemble des copies des dessins rele- vés. Marguerite Dellenbach lui répond rapidement (lettre du 10 avril 1939).

Elle le remercie, ainsi que Paul Rivet, pour cette réponse positive et lui signale qu'elle va demander l'autorisation aux différents musées possesseurs des bambous déjà étudiés de bien vouloir accepter l'envoi de ces copies. Elle doit cependant attendre le retour du professeur Eugène Pittard, directeur du musée, pour envoyer les copies des bambous de Genève et la confirmation comme quoi son institution prendra à sa charge les frais de transport des œuvres. Après plus d'un mois, elle peut lui confirmer l'envoi de six relevés de bambous gravés du musée de la Rochelle et de quatorze relevés du musée de Genève (lettre du 15 mai 1939). Elle lui annonce également l'envoi dans une dizaine de jours d'héliographies des relevés des bambous de Bâle et de Berne, mais lui dit aussi qu'elle reste dans l'attente de la réponse du musée Pigorini de Rome. Charles van den Broek remercie Marguerite Dellenbach pour ces héliographies qui sont bien arrivées et lui annonce l'envoi prochain d'un premier lot de bambous (lettre du 31 mai 1939). Il lui demande s'il doit les envoyer en port-dû ou en port-payé et en donne les valeurs d'assurance estimées à 600 Francs pièce selon l'expert Monsieur Ratton. Quinze jours après (lettre du 2 juin 1939), Marguerite Dellenbach accuse réception de cette lettre en le remerciant très vivement pour cet envoi tant attendu et en lui précisant de l'envoyer en port-payé avec une facture des frais d'envoi et d'assurance au nom du musée d'Ethnographie de Genève.

Un courrier de l'étude Gayet et Rémy, adressée à l'attention de monsieur Duchemin au musée de l'Homme (lettre du 29 juin 1939), confirme l'assurance " Tous risques », excepté le risque de guerre de treize bambous gravés auprès des Lloyd's de Londres pour un total de 9400 francs (huit bambous à 800 francs et cinq bambous à 600 francs). La nuance « excepté les risques de guerre » n'est pas anodine à cette époque ! Le 16 juillet 1939, une lettre de Marguerite Dellenbach à Charles van den Broek confirme la livraison des treize bambous par la maison Véron Grauer et Cie au musée d'Ethnographie de Genève. En remerciant Charles van den Broek de lui donner l'opportunité de pouvoir étudier, de visu, ces pièces, elle lui précise qu'elle va se hâter de les lui renvoyer. Mais Charles van den Broek repart vers le Pacifique à la fin du mois d'août, chargé d'une mission archéologique aux îles Marquises. Il laisse des consignes à son assistant Marioni pour gérer le département en son absence. La Seconde Guerre mondiale est déclarée le 3 septembre 1939, ce qui vient compliquer 
les échanges entre la France et la Suisse. Les bambous sont renvoyés à Paris et Charles van den Broek ne peut rentrer à Paris. Marguerite Dellenbach vient faire, en avril 1940, une conférence au musée de l'Homme. Le 10 février 1941, Paul Rivet doit quitter précipitamment Paris vers l'Amérique pour échapper à la vague d'arrestations visant les membres du réseau du musée de l'Homme qui viennent d'être dénoncés aux autorités allemandes. Charles van den Broek quitte Tahiti via la Nouvelle-Zélande pour rejoindre le général de Gaulle à Londres.

C'est en 1943 que Marguerite Dellenbach reprend contact avec le musée de l'Homme et son nouveau directeur, le professeur Vallois (lettre du 10 juin 1943), alors que la France est encore occupée. Elle souhaiterait pouvoir se procurer des photographies ou des calques des estampages et des relevés effectués par Marius Archambault sur les pétroglyphes de NouvelleCalédonie et déposés au laboratoire d'anthropologie du musée de l'Homme. Elle lui fait part de ses regrets que seuls un petit nombre de ces relevés ait été publié par G. H. Luquet. Ces intentions sont clairement exprimées par cette phrase :

« Nous serions extrêmement heureux de pouvoir mieux comprendre, grâce à ces documents d'Archambault, la lecture des bambous gravés et de pouvoir ainsi apporter une connaissance plus précise à la vie des Néo-Calédoniens. »

Elle propose que ces documents soient transmis éventuellement par Monsieur le Comte de Begouen, de Toulouse, qui lui a proposé dernièrement de lui prêter ses bambous gravés. Il est très probable que le professeur Vallois a transmis cette lettre à Maurice Leenhardt, puisque qu'une adresse de ce dernier - Mr Leenhardt, Fonfroide, route de Ganges, Montpellier - se trouve manuscrite au dos du courrier.

On ne trouve pas de trace de correspondance durant les quinze années où Marguerite Lobsiger-Dellenbach fut directrice du MEG. C'est peu de temps après la retraite de celle-ci, le $1^{\text {er }}$ août 1967, que Françoise Girard, chargée du département Océanie du musée de l'Homme, lui écrit pour lui demander conseil au sujet d'une écorce peinte australienne que lui propose pour achat Monsieur Cranley de Lausanne pour la somme de 2500 francs (lettre du 6 décembre 1967). Marguerite Lobsiger-Dellenbach lui confirme par courrier avoir vu cette pièce que le vendeur lui avait également présentée à Genève (lettre du 10 décembre 1967). Elle lui dit avoir refusé de faire cet achat car Karel Kupka (1972), qui se trouvait à Genève à ce moment, lui avait donné des informations qui avaient déterminé sa décision. Elle termine sa lettre en faisant part à son interlocutrice de sa satisfaction d'être retraitée et d'avoir ainsi retrouvé sa « liberté » tout en plaignant sincèrement son successeur, André Jeanneret, pour le travail qu'elle lui laisse.

En 1968, Marguerite Lobsiger-Dellenbach rédige une dernière liste des bambous gravés néo-calédoniens qu'elle a étudiés avec son époux. Sur un corpus de quatre-vingt-treize pièces décalquées et étudiées, seules cinquantequatre ont été publiées. Patrick O'Reilly (lettre du 12 mars 1953), qui entretint une riche correspondance avec le couple, lui suggère de publier l'ensemble de ses travaux sur les bambous gravés (Colombo Dougoud, 2008 : 30). Malheureusement, ce projet ne se concrétisa pas. Par ailleurs, le couple n'eut jamais l'opportunité de se rendre en Nouvelle-Calédonie. Ce qui est étonnant, car Marguerite Lobsiger-Dellenbach réalisa de nombreuses missions à l'étranger. Pourquoi ne se rendit-elle jamais sur le terrain pour confronter ses propres interprétations avec celles des Kanak, tenant des traditions locales ? Le Journal de la Société des Océanistes publia de 1951 à 1967 quatre articles consacrés aux bambous gravés et cosignés par le couple LobsigerDellenbach. Le premier (1951 : 105-121) s'intéresse à une collection privée alors que le second, dans le numéro spécial sur la NouvelleCalédonie (1953 : 229-243), décrit les aspects de la représentation des Européens par les Kanak. Le troisième (1958: 37-50) vient compléter l'étude des bambous gravés dans les collections des musées helvétiques. Le quatrième (1967: 59-99) est particulièrement intéressant pour nous, puisqu'il traite de la collection du MNAAO maintenant regroupée avec celle du musée de l'Homme au musée du quai Branly, qui se trouve ainsi avoir la plus importante collection de bambous gravés kanak au monde. Dans l'introduction de cet article, Marguerite Dellenbach remercie le professeur Jean Guiart pour avoir autorisé le prêt de ces bambous au musée de Genève pour qu'ils puissent être étudiés par ses soins. La correspondance à ce sujet doit faire partie des archives du MNAAO conservées au musée du quai Branly. La collection de bambous gravés du MNAAO se trouve maintenant regroupée avec celle du musée de l'Homme au musée du quai Branly, qui se trouve ainsi conserver le plus important ensemble de bambous gravés kanak au monde, soit soixante-dix pièces sur les cent cinquante existantes dans les collections des musées français, selon Roger Boulay ( $c f$. ci-dessus).

La dernière correspondance de Marguerite Lobsiger-Dellenbach avec le musée de l'Homme 
(lettre du 5 novembre 1970) montre que sa mise à la retraite n'a pas tari sa passion pour les bambous gravés. Dans celle-ci, elle demande à Françoise Girard des précisions au sujet d'une liste de numéros de bambous gravés qu'elle lui avait remise en juin 1965. Un des numéros des bambous étudiés en 1940 aurait été corrigé sur cette liste par Madame Clarac (une de ses collaboratrices). Elle conclut sa lettre ainsi :

« Le vieux couple Lobsiger ne se porte pas trop mal. Nous avons presque terminé l'étude des bambous de Leyden. »

Françoise Girard lui répond (lettre du 10 novembre 1970) en lui signalant qu'elle ne retrouve aucune trace d'une correspondance avec Madame Clarac et que le bambou en question est vraisemblablement celui portant le numéro de dépôt D.39.3.1871 acquis par les membres de l'expédition de La Korrigane en Nouvelle-Calédonie. C'est en effet, en 1935, que le révérend père Chevreuil de la mission de $\mathrm{La}$ Conception près de Nouméa fit don de ce bambou provenant de la collection de son prédécesseur le révérend père Pionnier ( $c f$. Guicciardi, p. 52, in Coiffier, 2001). Ce bambou gravé fut classé initialement sous le numéro MH.38.35.12 avant d'être mis par erreur sous un numéro de dépôt.

Cette correspondance, étalée sur plus de trente années, demeure un témoignage unique des relations entretenues entre des personnes qui ont marqué l'histoire des collections océaniennes européennes ; Charles van den Broek, Françoise Girard et, bien sûr, Marguerite LobsigerDellenbach.

\section{BIBLIOGRAPHIE}

COIFFIER Christian (éd.), 2001. Le voyage de La Korrigane dans les mers $d u$ Sud, Paris, Hazan/MnHN.

Colombo doudoug Roberta (éd.), 2008. Bambous kanak, une passion de Marguerite LobsigerDellenbach, Genève, Gollion-Infolio éditions, musée d'Ethnographie de Genève.

Grunevald R., 1936. Trois bambous gravés néocalédoniens, inédits, L'Anthropologie 46, pp. 633 640.

KupKa Karel, 1972. Peintres aborigènes d'Australie, Paris, Société des Océanistes, Publications de la Société des Océanistes 24.

Dellenbach Marguerite et Georges Lobsiger, 1938. Gravures sur bambous (Nouvelle-Calédonie), L'Ethnographie 35-36, pp. 35-49.

LOBSIGER-DELlENBACH Marguerite et Georges, 1951. Description de trois bambous gravés de NouvelleCalédonie de la collection de Monsieur Ratton, Journal de la Société des Océanistes VII, 7, pp. 105121.

_, 1953. Quelques aspects de la civilisation européenne gravés sur les bambous par les NéoCalédoniens, Journal de la Société des Océanistes IX, 9, pp. 229-243.

-, 1958. Deux bambous gravés de NouvelleCalédonie de la collection de Lausanne, Journal de la Société des Océanistes XIV, 14, pp. 37-50.

_, 1965. Architecture de Nouvelle-Calédonie (gravures sur bambou), in Carl A. Schmitz und Robert Wildhaber, Festschrift Alfred Bühler, Basel, PharosVerlag Hansrudolf Schwabe AG, Band 2, pp. 271287.

—, 1967. Dix bambous gravés néo-calédoniens du musée des Arts africains et océaniens (Paris), Journal de la Société des Océanistes XXIII, 23, pp. 59-99.

LuQueT C. H., 1926. L'art néo-calédonien, documents recueillis par Marius Archambault, Paris, Institut d'Ethnologie, coll. Travaux et mémoires.

VAN DEN Broek D’Obrenan, 1939. Le voyage de La Korrigane, Paris, Payot. 
